\title{
Snake circumvents constraints on prey size
}

\author{
Instead of swallowing a victim whole, crab-eating snakes tear off bite-sized pieces.
}
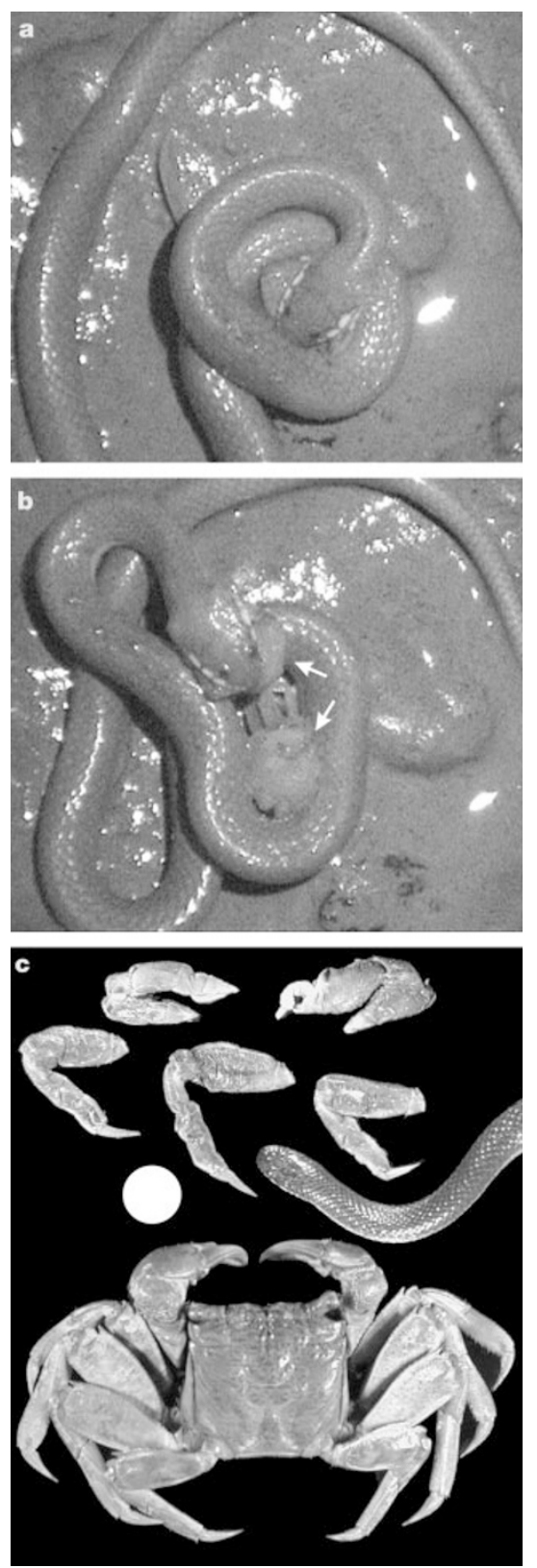

Figure 1 Feeding behaviour and prey size of the snake Gerarda prevostiana. a, b, Infrared video images of $G$. prevostiana $12.6 \mathrm{~s}$ before (a) and $0.6 \mathrm{~s}$ after (b) completely tearing apart the carapace of a freshly moulted grapsid crab that was considerably wider than the snake's head. Both pieces of the carapace (arrows) were consumed. For videos showing this behaviour, see www.biology.uc.edu/ faculty/jayne/gerarda_feed2.htm. c, Five appendages (top) from a freshly moulted crab (Episesarma versicolor) that was attacked and partially consumed by a 33-g G. prevostiana snake (middle) in the wild. The intact, 41-g E. versicolor shown at the bottom is similar in size to the crab that was attacked. The white circle (actual diameter, $13 \mathrm{~mm}$ ) is the maximum gape of the snake (determined by inserting cylindrical gauges with 1-mm diameter increments into the mouth of the anaesthetized snake). or or animals who are unable to take bites out of their food, the size of the food item that can be consumed is constrained by the maximal size of the mouth opening (gape) - snakes are an example of gape-limited predators and they usually swallow their prey whole ${ }^{1,2}$. Here we describe unique feeding behaviours in two closely related species of snake, which circumvent their gape limitation by removing and consuming pieces from newly moulted crabs that are too large to be swallowed intact. This evolutionary innovation is surprising, as the needle-like teeth and highly mobile bones that facilitate the capture and engulfment of large, whole prey by snakes are ill-suited both to cutting and to generating large bite forces.

We captured individuals of the homalopsine snake species Gerarda prevostiana and Fordonia leucobalia and examined their gut contents. We found that G. prevostiana contained the remnants of only freshly moulted crabs, whereas F. leucobalia contained material exclusively from hard-shelled crabs. F. leucobalia has several morphological ${ }^{3}$ and some behavioural ${ }^{4}$ specializations that are associated with its unusual diet. However, G. prevostiana, the diet and feeding behaviour of which have not previously been investigated, lacks the hypertrophied cranial musculature and short and blunt teeth of F. leucobalia ${ }^{5}$. We therefore collected G. prevostiana and a variety of crabs in Singapore and observed the snakes feeding in a dark room with infrared video cameras.

Captive $G$. prevostiana $(n=15)$ refused to attack hard-shelled crabs, but bit and rapidly swallowed freshly moulted crabs. In $85 \%$ of trials $(n=26)$, the snake pulled its head through a loop in its body and continued to pull on the crab while coiled around the animal (Fig. 1a, b). In some trials (35\%), snakes broke off and consumed up to four crab legs but none of the carapace. The carapace of the crab was either partially or completely torn apart in $20 \%$ and $32 \%$, respectively, of the 22 feedings in which snakes demonstrated the 'loop-and-pull' behaviour (Fig. 1a, b). Tearing of the carapace was most common for large crabs, and only occurred when a loop-and-pull action was used.

We also captured two G. prevostiana individuals that had consumed pieces of newly moulted crabs that were much larger relative to the snake than any of those used in laboratory trials (Fig. 1c). Consequently, ripping crabs apart is unlikely to be incidental, and probably allows $G$. prevostiana to regularly consume crabs with body sizes that exceed our estimate of its maximal gape.
These two species of crab-eating snake have not merely retained a common feeding mechanism of a crustacean-eating ancestor. The feeding mechanism used by captive F. leucobalia $(n=12)$ to eat both hard-shelled $(n=50)$ and soft-shelled $(n=6)$ crabs differed from that used by G. prevostiana in the mode of attacking prey, how prey was restrained, the usual orientation for swallowing the crab's body, and how pieces were torn from prey. In contrast, the patterns of coiling shown by many lineages of constricting snakes are usually evolutionarily conserved ${ }^{6}$. When F. leucobalia consumed hard-shelled crabs, the carapace always remained intact, contrary to a suggestion that this snake uses its jaws to crush $\mathrm{crabs}^{3}$. By using a method similar to that used for breaking legs off crabs $^{4}$, however, captive $F$. leucobalia did tear carapaces apart in $50 \%$ of trials with very large, soft-shelled crabs.

We are unaware of any other species of snake that regularly tears its prey apart so that it can consume larger prey than could be swallowed whole. The novel feeding mechanism used by $G$. prevostiana is surprising in view of its relatively unremarkable anatomy, which cautions against drawing functional inferences from anatomy in the absence of behavioural studies.

The diversity of tropical aquatic snakes in Singapore correlates well with their range of feeding mechanisms and specialisms. Within a single mangrove, F. leucobalia and G. prevostiana consume the same species of crab, but partition this resource by relying on different moult stages; two other species, Cantoria violacea and Cerberus rynchops, eat only snapping shrimp and fish, respectively ${ }^{7}$. Bruce C. Jayne ${ }^{\star}$, Harold K. Voris $\dagger$,

Peter K. L. Ng $\neq$

${ }^{*}$ Department of Biological Sciences, University of

Cincinnati, Ohio, 45221-0006, USA

e-mail: jaynebc@email.uc.edu

$\dagger$ Division of Amphibians and Reptiles, Field

Museum of Natural History, 1400 South Lake Shore Drive, Chicago, Illinois 60605, USA

$\ddagger$ Raffles Museum of Biodiversity Research,

Department of Biological Sciences, National

University of Singapore, Science Drive 4,

Singapore 119260

1. Cundall, D. \& Greene, H. W. in Feeding: Form, Function and Evolution in Tetrapod Vertebrates (ed. Schwenk, K.) 293-333 (Academic, New York, 2000).

2. Arnold, S. J. in Snakes: Ecology and Behavior (ed. Collins, J. T.) 87-115 (McGraw-Hill, New York, 1993).

3. Savitzky, A. H. Am. Zool. 23, 397-409 (1983).

4. Shine, R. \& Schwaner, T. Copeia 1985, 1067-1071 (1985).

5. Voris, H. K. et al. Copeia (in the press)

6. Greene, H. W. \& Burghardt, G. M. Science 200, 74-77 (1978)

7. Voris, H. K. \& Murphy, J. C. J. Nat. Hist. (in the press).

Competing financial interests: declared none. 